\title{
Molecular profiling leading to personalized treatment in breast cancer
}

\author{
Eva Valentina Klocker · Marija Balic · Günther Steger
}

Received: 6 October 2021 / Accepted: 16 December 2021 / Published online: 15 February 2022

(C) The Author(s) 2022

\begin{abstract}
Summary With the development of various targeted therapies in breast cancer, detection of biomarkers for predicting treatment efficacy gains importance. With the definition of intrinsic subtypes, breast cancer has paved the way for personalized treatment. Already known and recently recognized targets play an important role both in metastatic and early breast cancer. As a result, early molecular profiling is becoming a part of early diagnostic work-up. Notwithstanding, further treatment targets and agents are needed, particularly in the most aggressive subtype encompassing heterogenous diseases under the group of triple negative breast cancer.
\end{abstract}

Keywords Molecular signature - Targeted therapy · Biomarkers $\cdot$ Breast cancer $\cdot$ Next generation sequencing

Breast cancer is the most common tumor in women and the second most common cause for cancer-related death in women [1]. During the last decades, various treatment targets have been defined and have enabled efficient therapeutic strategies in breast cancer (BC). In order to offer the most suitable treat-

\footnotetext{
Dr. med. univ. E. V. Klocker $(\bowtie)$.

Assoz. Prof. PD Dr. med. univ. et scient. med. M. Balic Department of Oncology, Medical University of Graz, Auenbruggerplatz 15, 8036 Graz, Austria eva.klocker@medunigraz.at
}

Assoz. Prof. PD Dr. med. univ. et scient. med. M. Balic marija.balic@medunigraz.at

Univ.-Prof. Dr. G. Steger

Department of Internal Medicine I, Division of

Oncology, Medical University of Vienna, Waehringer

Guertel 18-20, 1090 Vienna, Austria

guenther.steger@meduniwien.ac.at ment, molecular profiling for already established biomarkers is needed. Various technologies have been established, covering a wide range of recognized targets along with potential further biomarkers, such as next generation sequencing (NGS) or ribonucleic acid(RNA)-based profiling or analyzing specific targets with polymerase chain reaction (PCR) based assays. In addition, well-preserved tumor tissue for testing is important. Defining treatment targets enables the administration of efficient agents beyond chemotherapy.

\section{Intrinsic subtypes and role of prognostic tests}

The classical stratification into the four subgroups luminal A, luminal B, human epidermal growth factor receptor 2 positive (HER2+) and triple negative is still crucial in the treatment of breast cancer. In addition to definition of the subtype, in hormone receptor positive (HR+), HER2- BC the estimation of risk for recurrence is important for treatment decisions. Several tools dealing with gene signatures have been developed to date. One of the signatures, PAM 50, estimates the intrinsic subtype by the gene signature and provides additional prognostic information [2]. Oncotype includes 21 genes for the prediction of the recurrence risk and enables the stratification into three groups: low, intermediate and high risk [3]. Sparrano et al. randomized 6711 patients with HR+, HER2-, lymph node negative $(\mathrm{N}-)$ breast cancer with an intermediate recurrence score of 11-25 between chemotherapy plus endocrine treatment and endocrine treatment alone in a prospective trial. In the total cohort no benefit from adding adjuvant chemotherapy in intermediate risk patients was shown. Nonetheless, women $\leq 50$ years old with a recurrence score of $16-25$ receiving chemotherapy in combination with endocrine 
therapy were more likely to show a benefit in diseasefree survival (DFS) [4].

\section{Challenging the definition of intrinsic subtypes}

\section{HER2 low}

The transmembrane tyrosine kinase receptor HER2 represents a well-established treatment target. HER2 positivity is defined by the American Society of Clinical Oncology/College of American Pathologist as immunohistochemistry (IHC)+++ [5]. In tissues with IHC++, meaning a weak to moderate membrane staining in $>10 \%$ of tumor cells, a reflex in situ hybridization (ISH) is recommended [5]. If tissue is IHC 0 or $\mathrm{IHC}+$, the result is HER2 negative (HER2-) [5]. However, HER2 low is IHC+ or IHC++ with a negative ISH result.

Due to the development of antibody-drug conjugates (ADCs), HER2 low has reached a new significance. ADC trastzumab-deruxtecan (T-Dx) shows effectiveness in HER2+, HER2 low and also in HER2 heterogeneous BC by the bystander killing effect [6-8]. The phase III trial was presented at ESMO 2021 and a significantly longer DFS and overall survival (OS) compared to trastuzumab-emtansine (T-DM1) in second line was detected in HER2+ mBC patients [9]. Phase III trials are ongoing in HER2 low patients.

Another second-generation antibody conjugate targeting HER2 is SYD958, called trastuzumab duocarmazine, with an ongoing phase III study TULIP, which showed promising results in the first analysis presented at ESMO 2021[10]. It showed efficacy in heavily pretreated, HER2+, HER2 low, and T-DM1 resistant breast cancers in a phase I trial [11].

\section{HER2 mutations}

Beyond HER2 low BC, ERB2 mutations remain a treatment challenge. HER2 mutations function as driver mutations in tumors without an amplification of HER2 too [12]. Tyrosine kinase inhibitors, such as HER2 targeting neratinib are able to directly inhibit intracellular signaling and have demonstrated their efficacy in HER2 mutations [13].

\section{HER3}

As HER2 needs another member of the ERB family to build heterodimers for signaling, human epidermal growth factor receptor 3 (HER3) plays a role in HER2+ breast cancers. HER3 appears to be a part of an escape mechanism in HER2 targeting treatments. As a result, HER3 and its mechanisms in signaling have been widely studied. There is evidence that HER3 is essential for the HER2 driven phosphatidylinositol 3-kinase-protein kinase B (PI3K-AKT) pathway by binding heterodimers with HER2 [14]. The ADC patritumab deruxtecan which specifically binds to HER3 is under evaluation [15]. Patritumab deruxtecan showed efficacy and tolerability in a phase 1 study including 21 pretreated metastatic breast cancer patients [16]. This ADC showed also promising results in a phase 1 study in nonsmall cell lung cancer, especially in patients with higher HER3 expression [17]. However, targeting HER3 in breast cancer is still a future goal and needs to be studied further.

\section{TNBC}

TNBC lacking estrogen receptor (ER)-, progesteron receptor $(\mathrm{PR})-$, and HER2 remains a treatment challenge. As shown by Burstein et al. and others, TNBC is a heterogeneous disease. Burstein et al. defined 4 TNBC subtypes using a PAM50 gene signature: luminal androgen receptor (LAR), mesenchymal (MES), basal-like immunosuppressed (BLIS) and basal-like immune activated (BLIA) [18]. Androgen-receptor (AR) might represent a treatment target, as AR- inhibitors were studied in AR expressing TNBC and showed clinical response [19]. The recently developed ADC sacituzumab-govitecan, targeting the antithrophoblast cell-surface antigen 2 (TROP2) has shown promising results in heavily pretreated patients with TNBC [20]. Further studies in triple negative and also HER2-BC are ongoing. To date, no selection by TROP2 expression is needed due to the bystander killing effect, but the efficacy is clearly correlated with the level of TROP2 expression.

\section{Potential of liquid biopsy}

Over the last decade, an increasing number of technologies to comprehensively analyze circulating deoxyribonucleic acid (DNA) and particularly the tumor fraction have evolved and now various possibilities for mutation testing are provided [21-23]. The phase 2a plasmaMATCH trial selected patients for specific treatment based on detection of mutations in circulating tumor DNA. The sensitivity and specificity in the circulating tumor DNA were comparable with mutation testing in tumor tissue for protein kinase B 1 (AKT1), HER2, and phosphoinositide-3-kinase, catalytic, alpha polypeptide (PIK3CA) mutations [21]. This trial paved the way for future personalized oncology approaches.

Another example in endocrine therapy-resistant breast cancers is the acquired estrogen receptor 1 (ESR1) mutation [24, 25]. The detection of ESR1 mutation in plasma after progression on aromatase inhibitor could be used for endocrine treatment decision [23]. Fulvestrant has shown efficacy in ESR1mutated breast cancer [23] and clinical trials are now testing the hypothesis in larger patient cohorts. 
Table 1 Therapeutic agents and their targets

\begin{tabular}{|c|c|c|}
\hline Target & Setting & Therapeutic agent \\
\hline Germline mBCRA1/2 & Adjuvant, palliative & PARP inhibitors \\
\hline HER2 & $\begin{array}{l}\text { Neoadjuvant, adjuvant, } \\
\text { palliative }\end{array}$ & $\begin{array}{l}\text { Trastuzumab, pertuzumab, } \\
\text { neratinib, lapatinib, tucatinib, T-DM1, T-Dx }\end{array}$ \\
\hline HER2 mutations & Palliative & Neratinib, lapatinib, tucatinib \\
\hline HER2 low/HER2 heterogeneity & In development palliative & T-Dx \\
\hline$H R$ & $\begin{array}{l}\text { Neoadjuvant, adjuvant, } \\
\text { palliative }\end{array}$ & SERM, Al \\
\hline PIKЗСА & Palliative & Alpelisib \\
\hline$P D-L 1 / C P S$ & Palliative, neoadjuvant? & Atezolizumab, pembrolizumab \\
\hline PTEN, AKT & Palliative? & AKT inhibitors? \\
\hline TROP2? & Palliative & Sacituzumab-govitecan \\
\hline
\end{tabular}

\section{Biomarkers with therapeutic relevance}

Besides the well-known HR and HER2 status, which became relevant during the 1990s, more biomarkers have gained therapeutic importance (Table 1).

\section{PIK3CA mutation}

The establishment of alpelisib in the therapy algorithm of PIK3CA-mutated metastatic HR+, HER2- BC requires testing for this mutation [26]. Although the PIK3CA mutation is an early mutation, an acquired PIK3CA mutation may occur under CDK4/6 inhibitor treatment associated with resistance to these drugs [24]. While the SOLAR trial demonstrated efficacy of alpelisib particularly in PIK3CA-mutated patients [27], in the BYlieve trial alpelisib showed a significant impact on progression free survival (PFS) after progress on CDK4/6 [22]. In this trial, PIK3CA mutation testing was done by NGS or PCR in circulating DNA in addition to tumor tissue [22].

\section{PI3K/AKT pathway}

Changes in the PI3K/AKT pathway like phosphatase and tensin homolog (PTEN) loss or hyperactivated AKT represent potentially useful treatment targets [28]. The PI3K/AKT signaling pathway plays an important role in breast cancer. PTEN possesses an inhibiting role, whereas AKT holds an activating function. The AKT inhibitors capivasertib and ipatasertib showed promising results in the phase II trials LOTUS and FAKTION $[29,30]$. However, the phase III trial IPATunity130 with ipatasertib in combination with paclitaxel in metastatic TNBC (mTNBC) failed to confirm its benefit [31]. A biomarker analysis of the IPATunity130 is planned and may provide additional information about the subgroups. However, the first results of the phase III trial analyzing capivasertib in this setting are not yet available.

\section{BRCA 1/2 mutation}

In HER2- disease, PARP inhibitors represent an active agent in germline-mutated BRreat CAncer gene (BRCA1/2) mBC [32, 33]. In mBC PARP inhibitors significantly prolong PFS [32, 33]. No significant impact in overall survival (OS) has been be detected. In ovarian cancer these agents are effective in both germline and somatic mutations [34]. However, the role of somatic BRCA1/2 mutations or "BRCAness" in $\mathrm{BC}$ is not fully clarified to this date. In the GeparOLA trial, for example, germline and somatic BRCA1/2 and additional mutations leading to homologous recombinant deficiency were included [35]. The effect of olaparib as an additional neoadjuvant agent was superior only in germline-mutated BRCA1/2 patients in the $\mathrm{HR}+$ and in the $<40$ years subgroups [35]. Another trial including germline mutations, somatic mutations, BRCA1/2 methylation status and BRCA $1 / 2$ messenger RNA (mRNA) analyzing the effect of carboplatin versus docetaxel in metastatic setting showed an effect of carboplatin solely in the germline-mutated cohort [36]. Nonetheless, only a small number of patients revealed a germline mutation in this study. The significance of somatic BRCA1/2 mutations or a "BRCAness type" for treatment in BC has not yet been confirmed. Further studies are needed, and one of the trials is the ABCSG45 neoadjuvant phase II study evaluating the combination of carboplatin and olaparib versus standard neoadjuvant chemotherapy in patients with "BRCAness". Germline BCRA1/2 mutation testing is becoming an imperative for TNBC and high-risk hormone receptor positive BC patients in the adjuvant setting based on the adjuvant OlympiaD trial. Administration of olaparib for 1 year after finishing adjuvant/neoadjuvant treatment in high risk germline-mutated BRCA1/2 patients leads to an improved invasive disease-free survival (iDFS) [37]. 


\section{PD-L1 and CPS score}

A clinically established biomarker beyond germline BRCA1/2 mutation status for treatment decision in TNBC is the programmed death-ligand 1 (PD-L1) status $[38,39]$. In mTNBC the addition of atezolizumab to nab-paclitaxel significantly prolonged PFS and improved median OS in PD-L1 positive patients [38]. PDL1 positivity was defined as PD-L1 expression in immune cells that covers $\geq 1 \%$ of the tumor and was assessed using the SP142 PD-L1 immunohistochemical assay (Ventana) [38]. In the KEYNOTE-355, the addition of pembrolizumab improved PFS in patients with a combined positive score (CPS) $\geq 10$ [39]. In contrast, the role of the PD-L1 status in the neoadjuvant setting is less prominent, since the benefit of pembrolizumab or atezolizumab was shown independently from the PD-L1 status [40, 41].

To sum up, with the development of novel targeted therapies, molecular profiling is gaining in importance. Whereas an increasing number of molecularly driven clinical trials is becoming available, it is increasingly difficult for clinical trials to represent the heterogeneity of breast cancer biology. Nevertheless, in today's clinical practice biomarker testing, it is a prerequisite to adequately treat breast cancer patients. Analysis of established biomarkers in BC is essential for all patients where treatment is available. Currently, it is still recommended to perform biopsies of new lesions in metastatic patients. The hope is that with the increasing number of studies, cell free DNA based mutation testing will demonstrate its reliability and robustness and at least partially replace the more invasive tissue testing. With the available novel technologies, the hope is to identify an increasing number of treatment targets and agents which are urgently needed especially for TNBC.

Funding Open access funding provided by Medical University of Graz.

Conflict of interest E.V. Klocker received congress fees from Pierre Fabre, Roche and Daiichi-Sankyo and congress fees from Novartis and AstraZeneca. M. Balic received honoraria and congress fees from Amgen, AstraZeneca, Bayer, Celgene, Daichii Sankyo, Pfizer, EliLilly, Novartis, Roche, MSD, Samsung, Pierre Fabre and Seagen. G. Steger declares that he has no competing interests.

Open Access This article is licensed under a Creative Commons Attribution 4.0 International License, which permits use, sharing, adaptation, distribution and reproduction in any medium or format, as long as you give appropriate credit to the original author(s) and the source, provide a link to the Creative Commons licence, and indicate if changes were made. The images or other third party material in this article are included in the article's Creative Commons licence, unless indicated otherwise in a credit line to the material. If material is not included in the article's Creative Commons licence and your intended use is not permitted by statutory regulation or exceeds the permitted use, you will need to obtain permission directly from the copyright holder. To view a copy of this licence, visit http://creativecommons.org/licenses/by/4.0/.

\section{References}

1. Siegel RL, Miller KD, Fuchs HE, et al. Cancer statistics, 2021. CACancerJClin. 2021;71(1):7-33. https://doi.org/10.3322/ caac.21654. Erratumin: CACancer JClin. 2021;71(4):359.

2. Parker JS, Mullins M, Cheang MCU, et al. Supervised risk predictor of breast cancer based on intrinsic subtypes. JClin Oncol. 2009;27(8):1160-7.

3. Paik S, Kim C, Baehner FL, et al. A Multigene assay to predict recurrence of Tamoxifen-treated, node-negative breast cancer. NEngl J Med. 2004;351(27):2817-26.

4. Sparano JA, Gray RJ, Makower DF, et al. Adjuvant chemotherapy guided by a 21-gene expression assay in breast cancer. NEngl J Med. 2018;379(2):111-21.

5. Wolff AC, Hammond MEH, Allison KH, et al. Human epidermal growth factor receptor 2 testing in breast cancer: AmericanSociety ofClinicalOncology/College of American Pathologists Clinical practice guideline focused update. JClin Oncol. 2018;36(20):2105-22.

6. Modi S, Saura C, Yamashita T, et al. Trastuzumab Deruxtecan in previously treated HER2-positive breast cancer. NEngl J Med. 2020;382(7):610-21.

7. Ogitani Y, Hagihara K, Oitate M, et al. Bystander killing effect of DS-8201a, a novel anti-human epidermal growth factor receptor 2 antibody-Drug conjugate, in tumors with human epidermal growth factor receptor 2 heterogeneity. Cancer Sci. 2016;107(7):1039.

8. Modi S, Park H, Murthy RK, et al. Antitumor activity and safety of Trastuzumab Deruxtecan in patients with HER2-low-expressing advanced breast cancer: results from a phase Ib study. JClin Oncol. 2020;38(17):1887.

9. Cortes J, Kim S, Chung W, et al. Trastuzumab deruxtecan (T-DXd) vs trastuzumab emtansine (T-DM1) in patients (Pts) with HER2+ metastatic breast cancer (mBC): Results of the randomized phase III DESTINY-Breast03 study. Ann Oncol. 2021;32(suppl_5):S1283-S346. https://doi.org/10. 1016/annonc/annonc741. abstrLBAl.

10. Manich SC, O'Shaughnessy J, Aftimos PG, et al. Primary outcome of the phase III SYD985.002/TULIP trial comparing [vic-] trastuzumab duocarmazine to physician's choice treatment in patients with pre-treated HER2-positive locally advanced or metastatic breast cancer. Ann Oncol. 2021;32(suppl_5):S1283-S346. https://doi.org/10.1016/ annonc/annonc741. abstr LBA15.

11. Banerji U, van Herpen CML, Saura C, et al. Trastuzumab duocarmazine in locally advanced and metastatic solid tumours and HER2-expressing breast cancer: a phase 1 dose-escalation and dose-expansion study. Lancet Oncol. 2019;20(8):1124-35.

12. Bose R, Kavuri SM, Searleman AC, et al. Activating HER2 mutations in HER2 gene amplification negative breast cancer. Cancer Discov. 2013;3(2):224-37.

13. Hyman DM, Piha-Paul SA, Won H, et al. HER kinase inhibition in patients with HER2- and HER3-mutant cancers. Nature. 2018;554(7691):189-94.

14. Dey N, Williams C, Leyland-Jones B, et al. A critical role for HER3 in HER2-amplified and non-amplified breast cancers: function of a kinase-dead RTK. Am J Transl Res. 2015;7(4):733-50.

15. Pascual T, Oliveira M, Ciruelos E, et al. SOLTI-1805 TOTHER3 study concept: a window-of-opportunity trial of Patritumab Deruxtecan, a HER3 directed antibody drug conjugate, in patients with early breast cancer. Front Oncol. 2021;11:638482.

16. Kogawa T, Yonemori K, Masuda N, et al. Single agent activity of U3-1402, a HER3-targeting antibody-drug conjugate, in 
breast cancer patients: Phase 1 dose escalation study. J Clin Oncol. 2018;36(15_suppl):2512-2512.

17. Janne PA, Baik C, Su WC, et al. Efficacy and safety of Patritumab Deruxtecan (HER3-DXd) in EGFR inhibitor-resistant, EGFR-mutated non-small cell lung cancer. Cancer Discov. 2021;2022 Jan;12(1):74-89.

18. Burstein MD, Tsimelzon A, Poage GM, et al. Comprehensive genomic analysis identifies novel subtypes and targets of triple-negative breast cancer. Clin Cancer Res. 2015;21(7):1688-98.

19. Traina TA, Miller K, Yardley DA, et al. Enzalutamide for the treatment of androgen receptor-expressing triple-negative breast cancer. JClin Oncol. 2018;36(9):884-90.

20. BardiaA, HurvitzSA, TolaneyAM, etal. Sacituzumab govitecan in metastatic triple-negative breast cancer. N Engl J Med. 2021;384:1529-41.

21. Turner NC, Kingston B, Kilburn LS, et al. Circulating tumour DNA analysis to direct therapy in advanced breast cancer (plasmaMATCH): a multicentre, multicohort, phase 2a, platform trial. Lancet Oncol. 2020;21(10):1296-308.

22. Rugo HS, Lerebours F, Ciruelos E, et al. Alpelisib plus fulvestrant in PIK3CA-mutated, hormone receptor-positive advanced breast cancer after a CDK4/ 6 inhibitor (BYLieve): one cohort of a phase 2, multicentre, open-label, noncomparative study. Lancet Oncol. 2021;22(4):489-98.

23. Fribbens C, O'Leary B, Kilburn L, et al. Plasma ESR1 mutations and the treatment of estrogen receptor-positive advanced breast cancer. JClin Oncol. 2016;34(25):2961-8.

24. O'Leary B, Cutts RJ, Liu Y, et al. The genetic landscape and clonal evolution of breast cancer resistance to Palbociclib plus Fulvestrant in the PALOMA-3 trial. Cancer Discov. 2018;8(11):1390-403.

25. Razavi P, Chang MT, Xu G, et al. The genomic landscape of endocrine-resistant advanced breast cancers. Cancer Cell. 2018;34(3):427-438.e6.

26. AndréF, Ciruelos E, Rubovszky G, et al. Alpelisibfor PIK3CAmutated, hormone receptor-positive advanced breast cancer. NEngl J Med. 2019;380(20):1929-40.

27. André F, Ciruelos EM, Juric D, et al. Alpelisib plus fulvestrant for PIK3CA-mutated, hormone receptor-positive, human epidermal growth factor receptor-2-negative advanced breast cancer: final overall survival results from SOLAR-1. Ann Oncol. 2021;32(2):208-17.

28. Lin J, Sampath D, Nannini MA, et al. Targeting activated Akt with GDC-0068, a novel selective akt inhibitor that is efficacious in multiple tumor models. Clin Cancer Res. 2013;19(7):1760-72.

29. Jones RH, Casbard A, Carucci M, et al. Fulvestrant plus capivasertib versus placebo after relapse or progression on an aromatase inhibitor in metastatic, oestrogen receptor-positive breast cancer (FAKTION): a multicentre, randomised, controlled, phase 2 trial. Lancet Oncol. 2020;21(3):345-57.

30. Kim SB, Dent R, Im SA, et al. Ipatasertib plus paclitaxel versus placebo plus paclitaxel as first-line therapy for metastatic triple-negative breast cancer (LOTUS): a multicentre, randomised, double-blind, placebo-controlled, phase 2 trial. Lancet Oncol. 2017;18(10):1360-72.

31. Dent R, Kim SB, Oliveira M, et al. Double-blind placebo-controlled randomized phase III trial evaluating first-line ipatasertib combined with paclitaxel for -PIK3CA/AKT1/PTEN-altered locally advanced unresectable or metastatic triple-negative breast cancer:
Primary results from IPATunity130 Cohort A. Cancer Res. 2021;81(4_Suppl):abstrGS3-04. SABCS20-GS3-04.

32. Robson M, Im SA, Senkus E, et al. Olaparib for metastatic breast cancer in patients with a Germline BRCA mutation. NEngl J Med. 2017;377(6):523-33.

33. Litton JK, Rugo HS, Ettl J, et al. Talazoparib in patients with advanced breast cancer and a Germline BRCA mutation. NEngl J Med. 2018;379(8):753-63.

34. Tomao F, Bardhi E, Di Pinto A, et al. Parp inhibitors as maintenance treatment in platinum sensitive recurrent ovarian cancer: an updated meta-analysis of randomized clinical trials according to BRCA mutational status. Cancer TreatRev. 2019;80:101909.

35. Fasching PA, Link T, Hauke J, Seither F, Jackisch C, Klare P, et al. Neoadjuvant paclitaxel/olaparib in comparison to paclitaxel/carboplatinum in patients with HER2-negative breast cancer and homologous recombination deficiency (GeparOLAstudy). Ann Oncol. 2021;32(1):49-57.

36. Tutt A, Tovey H, Cheang MCU, Kernaghan S, Kilburn L, Gazinska P, et al. Carboplatin in BRCA1/2-mutated and triple-negative breast cancer BRCAness subgroups: the TNT Trial. NatMed. 2018;24(5):628-37.

37. Tutt ANJ, Garber JE, Kaufman B, Viale G, Fumagalli D, Rastogi P, et al. Adjuvant Olaparib for patients with BRCA1- or BRCA2-mutated breast cancer. N Engl J Med. 2021;384(25):2394-405.

38. Schmid P, Rugo HS, Adams S, et al. Atezolizumab plus nabpaclitaxel as first-line treatment for unresectable, locally advanced or metastatic triple-negative breast cancer (IMpassion130): updated efficacy results from a randomised, double-blind, placebo-controlled, phase 3 trial. Lancet Oncol. 2020;21(1):44-59.

39. Cortes J, Cescon DW, Rugo HS, et al. Pembrolizumab plus chemotherapy versus placebo plus chemotherapy for previously untreated locally recurrent inoperable or metastatic triple-negative breast cancer (KEYNOTE-355): a randomised, placebo-controlled, double-blind, phase 3 clinical trial. Lancet. 2020;396(10265):1817-28.

40. Schmid P, Cortes J, Pusztai L, et al. Pembrolizumab for early triple-negative breast cancer. N Engl J Med. 2020;382(9):810-21.

41. MittendorfEA, Zhang H, Barrios $\mathrm{CH}$, et al. Neoadjuvant atezolizumab in combination with sequential nab-paclitaxel and anthracycline-based chemotherapy versus placebo and chemotherapy in patients with early-stage triple-negative breast cancer (IMpassion031): a randomised, doubleblind, phase 3 trial. Lancet. 2020;396(10257):1090-100.

Publisher's Note Springer Nature remains neutral with regard to jurisdictional claims in published maps and institutional affiliations.

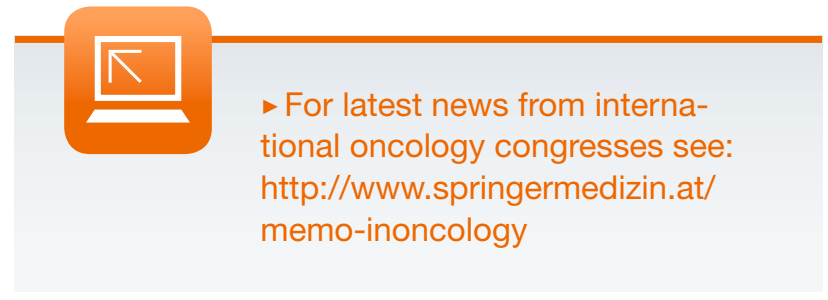

